OPEN ACCESS

Edited by:

Rudolf Lucas,

Augusta University, United States

Reviewed by:

Ralf Jacob,

University of Marburg, Germany

Hakon Leffler

Lund University, Sweden

*Correspondence:

Ah-Mee Park

ampk@med.kindai.ac.jp

Specialty section:

This article was submitted to

Inflammation,

a section of the journal

Frontiers in Immunology

Received: 15 May 2020 Accepted: 02 September 2020

Published: 23 September 2020

Citation:

Park A-M, Khadka S, Sato $F$ Omura S, Fujita M, Hsu DK, Liu F-T and Tsunoda I (2020) Galectin-3 as a Therapeutic Target for NSAID-Induced Intestinal UIcers.

Front. Immunol. 11:550366. doi: 10.3389/fimmu.2020.550366

\section{Galectin-3 as a Therapeutic Target for NSAID-Induced Intestinal Ulcers}

\author{
Ah-Mee Park ${ }^{1 *}$, Sundar Khadka', Fumitaka Sato', Seiichi Omura', Mitsugu Fujita', \\ Daniel K. Hsu' ${ }^{2}$, Fu-Tong Liu' ${ }^{2,3}$ and Ikuo Tsunoda ${ }^{1}$ \\ ${ }^{1}$ Department of Microbiology, Faculty of Medicine, Kindai University, Osaka, Japan, ${ }^{2}$ Department of Dermatology, University \\ of California Davis Health System, Sacramento, CA, United States, ${ }^{3}$ Institute of Biomedical Sciences, Academia Sinica, \\ Taipei, Taiwan
}

Non-steroidal anti-inflammatory drugs (NSAIDs) induce ulcers in the gastrointestinal tract, including the stomach and small intestine. NSAID-induced gastric ulcers can be prevented by taking acid-neutralizing/inhibitory drugs and cytoprotective agents. In contrast, there are no medicines to control NSAID-induced small intestinal ulcers, which are accompanied by a mucosal invasion of bacteria and subsequent activation of immune cells. Galectin-3 (Gal3), an endogenous lectin, has anti-microbial and proinflammatory functions. In the small intestine, since Gal3 is highly expressed in epithelial cells constitutively and macrophages inducibly, the Gal3 level can affect microbiota composition and macrophage activation. We hypothesized that the modulation of Gal3 expression could be beneficial in NSAID-induced intestinal ulcers. Using Gal3 knockout (Gal3KO) mice, we determined whether Gal3 could be a therapeutic target in NSAIDinduced intestinal ulcers. Following the administration of indomethacin, an NSAID, we found that small intestinal ulcers were less severe in Gal3KO mice than in wild-type (WT) mice. We also found that the composition of intestinal microbiota was different between WT and Gal3KO mice and that bactericidal antibiotic polymyxin B treatment significantly suppressed NSAID-induced ulcers. Furthermore, clodronate, a macrophage modulator, attenuated NSAID-induced ulcers. Therefore, Gal3 could be an exacerbating factor in NSAID-induced intestinal ulcers by affecting the intestinal microbiota population and macrophage activity. Inhibition of Gal3 may be a therapeutic strategy in NSAID-induced intestinal ulcers.

Clinical Trial Registration: www.ClinicalTrials.gov, identifier NCT03832946.

\footnotetext{
Keywords: adverse effect, animal model, cyclooxygenase-2 inhibitors, microbiome, PAS stain, small intestine, $16 \mathrm{~S}$ rRNA, gastrointestinal flora
}

\section{INTRODUCTION}

Although non-steroidal anti-inflammatory drug (NSAID) therapy has been used to suppress inflammation and pain, it sometimes induces potentially life-threatening complications related to NSAID-induced gastrointestinal ulcers (1-3). In the stomach, although NSAID-induced ulcers are occasionally fatal, it can be prevented/treated by taking acid-neutralizing/inhibitory drugs and cytoprotective agents. In the small intestine, NSAIDs can also induce ulcers, resulting in bleeding 
and perforation $(1,3)$. Unlike the stomach ulcers, there is no treatment to control NSAID-induced small intestinal ulcers.

Galectin-3 (Gal3) is one of the galectin family members, which is highly expressed by activated macrophages as well as various cell types constitutively including gastrointestinal epithelial cells $(4,5)$. Gal3 has been shown to have a variety of pro-inflammatory and anti-microbial functions (6-11). For example, activated macrophages have been shown to express Gal3, which plays roles in not only the survival and phagocytosis of macrophages/neutrophils (6-8), but also neutrophil extravasation (9-11). Gal3 can be directly bacteriostatic for Helicobacter pylori (12), Staphylococcus pneumonia (9), and cytocidal for Candida albicans (13). On the other hand, Gal3 is known to be an exacerbating factor in several diseases experimentally and clinically, including idiopathic pulmonary fibrosis $(14,15)$, non-alcoholic steatohepatitis with cirrhosis (16), and ovarian carcinoma (17). Thus, Gal3 is considered as a therapeutic target for these diseases (18), in which the development of Gal3 inhibitors has been attempted.

Non-steroidal anti-inflammatory drugs-induced small intestinal ulcers have been proposed to develop with several factors: a decrease in mucus secretion caused by low prostaglandin synthesis; the mucosal invasion of bacteria; and activation of immune cells including macrophages $(19,20)$. Since Gal3 has pro-inflammatory and anti-microbial functions, changes in the Gal3 levels can affect immune cell activation and bacterial composition in the intestine. Here, we hypothesize that the modulation of Gal3 expression can be beneficial in NSAID-induced intestinal ulcers. In the following sections, we will introduce our experimental findings, in which small intestinal ulcers were suppressed in Gal3 knockout (Gal3KO) mice following administration of indomethacin (Indo), an NSAID. We will propose that the inhibition of Gal3 can be a therapeutic strategy in NSAID-induced intestinal ulcers.

\section{GALECTIN-3 IN INTESTINAL ULCERS}

\section{Attenuation of NSAID-Induced Small Intestinal Ulcers in Gal3KO Mice}

We first examined Gal3 expression in the small intestine (Figure 1A) in 10-14 week-old wild-type (WT) CD1 mice (Charles River Laboratories Japan, Yokohama, Japan) and Gal3KO CD1 mice (12). In WT mice, enterocytes of the small intestine moderately expressed Gal3 in the cytoplasm. On the other hand, mononuclear cells in the lamina propria (LP) and subepithelial dome region (SED) of the Peyer's patch (PP) highly expressed Gal3. We confirmed that Gal3KO mice had no Gal3 expression. Although Gal3 has been reported to play a role in protein trafficking and morphogenesis of enterocytes of the small intestine (21), we found no obvious morphological changes in the small intestine of Gal3KO mice. We also assessed the intestinal mucus level with periodic acid-Schiff (PAS) stain, by which mucus is stained purple-magenta (Figure 1B). PASpositive mucus was observed in the cytoplasm of goblet cells and the luminal surface of the enterocytes. We found similar numbers of goblet cells and thickness of PAS-positive mucus in WT and Gal3KO mice.

Experimentally, a mouse model for small intestinal ulcers has been induced with oral administration of Indo to conventionally fed mice without fasting; this regimen does not induce ulcers in the stomach (22). To examine the roles of Gal3 in the small intestine, we administrated Indo to WT and Gal3KO mice, harvested the gastrointestinal tissues, and identified ulcers macroscopically. We detected ulcers predominantly in the jejunum, but not in the ileum; there was no evident ulcer in the stomach or colon of these mice, which was most likely due to the low dosage of Indo administered to conventionally fed (no-fasted) mice (22). We quantified the severity of ulcers in the jejunum using the ulcer score (23), and found that Gal3KO mice had significantly lower ulcer scores than WT mice (Figures 2A,B). Microscopically, we found severe ulceration in the WT mice treated with Indo (WT+Indo) (Figure 2C). Although $\mathrm{F} / 80^{+}$macrophages were detected in the lamina propria in all mice, $\mathrm{F} 4 / 80^{+}$macrophages were increased only in the ulcerated lesions of the WT+Indo. $\mathrm{Ly}_{6} \mathrm{G}^{+}$neutrophils were accumulated in the ulcerated lesions of the WT+Indo but not detectable in the control WT mice or the Gal3KO mice treated with Indo (Gal3KO+Indo). We also assessed the severity of ulcers by the fecal occult blood (FOB) levels. We found that the FOB levels were significantly lower in the Gal3KO+Indo mice than in the WT+Indo mice, which were associated with the ulcer scores (Figure 3). Without Indo administration, FOB was not detected.

\section{Role of Microbiota in NSAID-Induced Ulcers in WT and Gal3KO Mice}

Indomethacin is inactivated in the liver by glucuronidation and excreted in bile, and then, in the intestine, glucuronidesIndo are cleaved by bacterial $\beta$-glucuronidase, releasing free Indo. Subsequently, the enterocytes are exposed to relatively high concentrations of free Indo (17). Bacterial $\beta$ glucuronidase activities differ amongst bacterial species (24); several intestinal bacteria species in the families Lachnospiraceae and Bacteroidaceae have $\beta$-glucuronidase. Inhibiting the $\beta$-glucuronidase activity has been reported to protect mice against Indo-induced small intestinal ulcers (25). Thus, we compared the $\beta$-glucuronidase activities of fecal samples between WT and Gal3KO mice. We found no significant differences between the two mouse groups (Figure 4), suggesting that the $\beta$-glucuronidase levels are irrelevant to the suppression of Indo-induced ulcers in Gal3KO mice.

Non-steroidal anti-inflammatory drug-induced intestinal ulcers are known to occur by the mucosal invasion of intestinal bacteria $(25,26)$. Thus, we harvested small intestinal contents from WT and Gal3KO mice, isolated bacterial DNA, and conducted a $16 \mathrm{~S}$ rRNA-based microbiome analysis. We compared the intestinal microbiomes between naïve WT and Gal3KO mice. At the family level, the intestinal microbiomes were mainly composed of the family Lactobacillaceae in both WT and Gal3KO mice (Supplementary Figure 1). Other than Lactobacillaceae, the families S24-7 and Bifidobacteriaceae were highly abundant in WT mice; the Clostridiales order unknown 
A

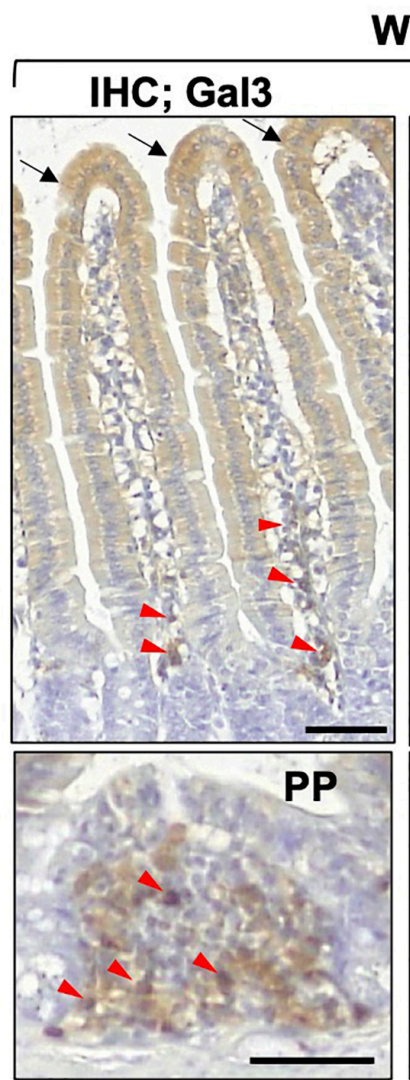

WT

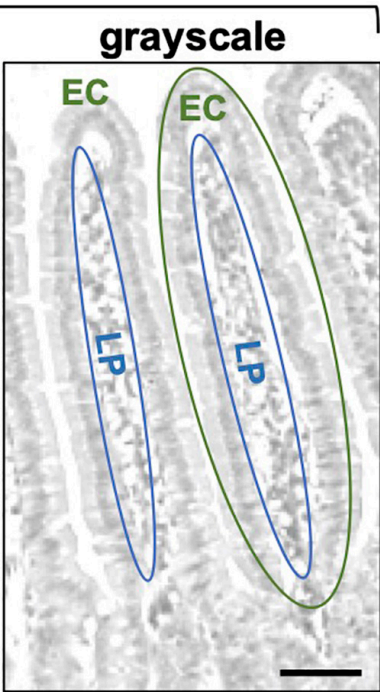

Gal3KO
IHC; Gal3
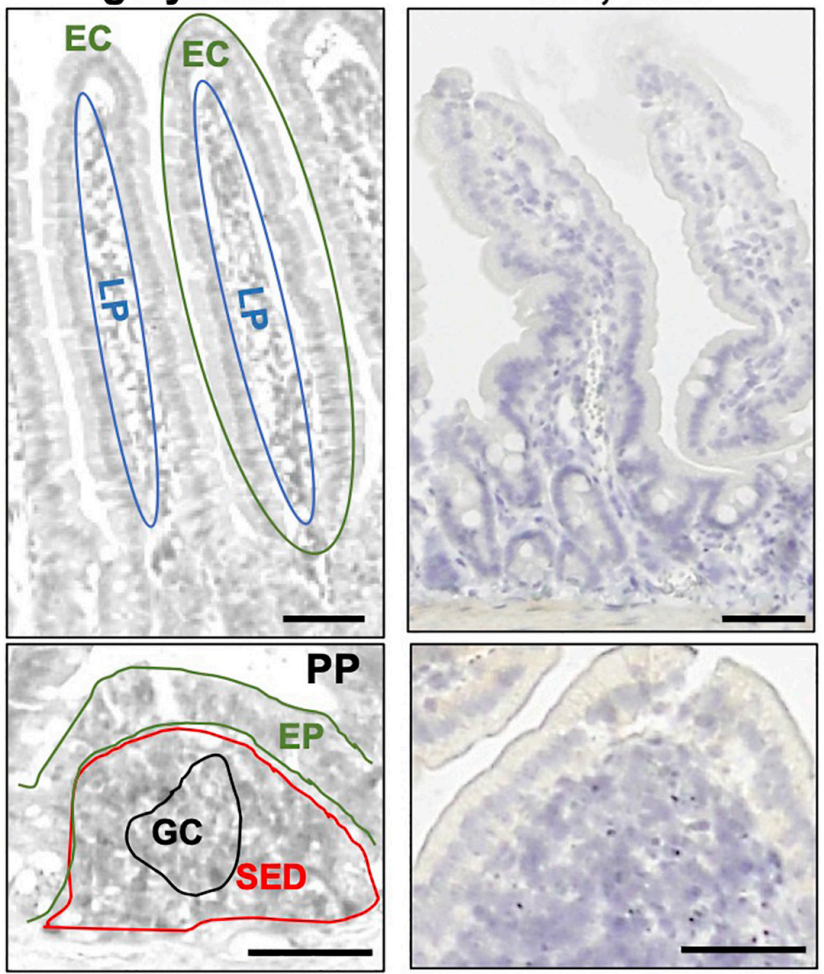

Bar $=\mathbf{5 0} \boldsymbol{\mu m}$

B PAS staining
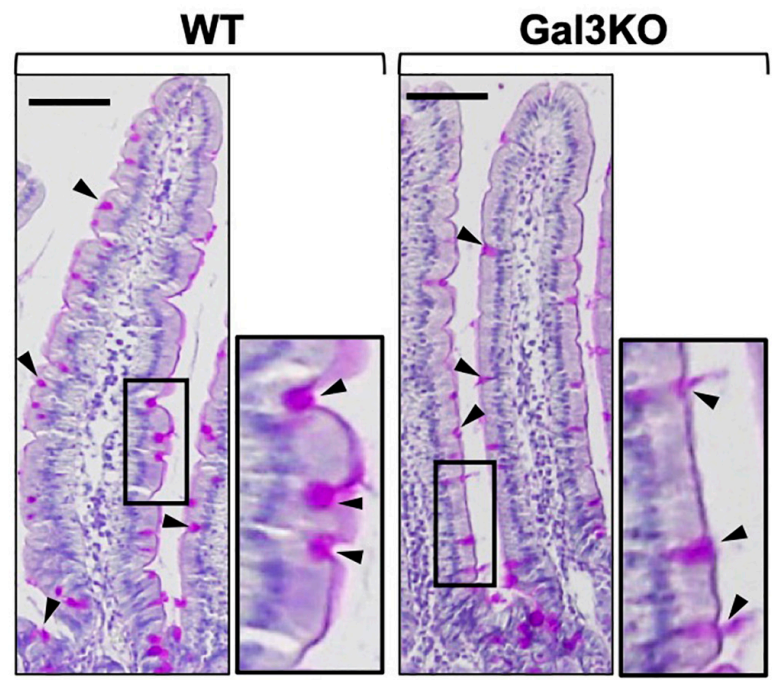

Bar $=\mathbf{5 0} \boldsymbol{\mu m}$

FIGURE 1 | Galectin-3 (Gal3) and mucin staining. (A) We conducted immunohistochemistry with anti-Gal3 antibody (BioLegend, San Diego, CA, United States) using a Histofine SAB-PO kit (Nichirei Biosciences; Tokyo, Japan), in 4- $\mu$ m thick small intestine sections of wild-type (WT) and Gal3 knockout (Gal3KO) mice. Gal3 was stained brown, and nuclei were counterstained with hematoxylin (blue). In WT mice, we found moderate Gal3 staining in the cytoplasm of enterocytes (EC, black arrows) and intense staining of mononuclear cells (red arrowheads) in the lamina propria (LP). In the Peyer's patch (PP), Gal3 positive cells were detected in the subepithelial dome region (SED), but not in the germinal center (GC). Gal3KO mice had no Gal3 positive cells. The middle grayscale panels were shown to indicate anatomical structures. EP, epithelium. (B) Periodic acid-Schiff (PAS) staining of intestine sections of WT and Gal3KO mice. The cytoplasm of goblet cells (black arrowheads) and the luminal surface of enterocytes were stained purple-magenta due to the presence of mucins. 


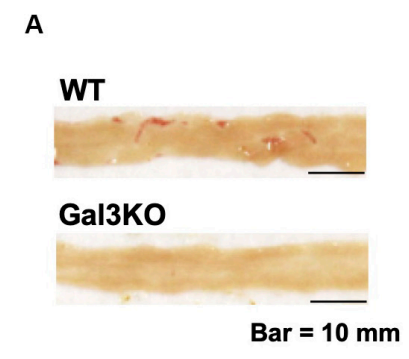

B

C

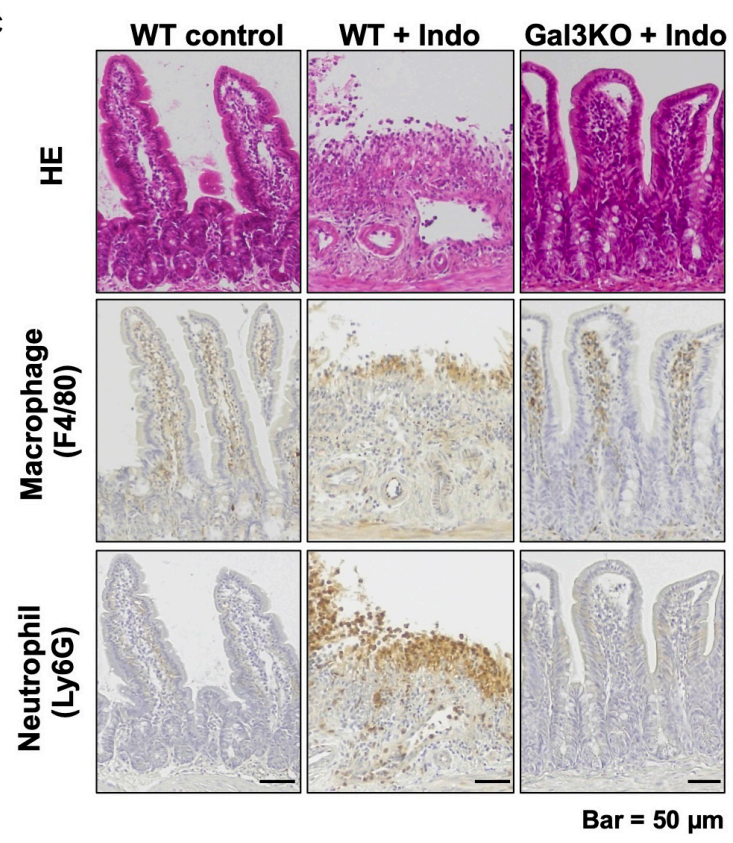

FIGURE 2 | Indomethacin (Indo)-induced small intestinal ulcers. We treated WT and Gal3KO mice with Indo, and $18 \mathrm{~h}$ later, harvested the small intestines ( $n=7$ per group). We determined the Indo concentration $5 \mathrm{mg} / \mathrm{kg}$ body weight by "Human equivalent dose calculation" (42). Indo (FUJIFILM Wako, Osaka, Japan) was solved in a $0.5 \% \mathrm{NaHCO}_{3}$ solution and administrated to non-fasted mice by using a stomach tube. (A) We opened the small intestine along the anti-mesenteric side, and took macroscopic images with a scale by a digital camera (Canon, Tokyo, Japan). Representative macroscopic images of the jejunum from WT (ulcer score $=3$ ) and Gal3KO mice (ulcer score $=0$ ). $\mathrm{Bar}=10 \mathrm{~mm}$. (B) The ulcer severity was assessed using the ulcer score (23) with modification. The macroscopic ulceration areas were captured and quantified by an Image J software (NIH, Bethesda, MD, United States) and summed per jejunum. The entire jejunum areas were similar in all mice examined and were around $1,200 \mathrm{~mm}^{2}$. We used the modified ulcer score as follows: $0=$ no lesion, $1=$ less than $5 \mathrm{~mm}^{2}, 2=5 \sim 20 \mathrm{~mm}^{2}, 3=21 \sim 40 \mathrm{~mm}^{2}$, $4=41 \sim 70 \mathrm{~mm}^{2}$, and $5=$ more than $71 \mathrm{~mm}^{2} .{ }^{*} P<0.05$ by the Mann-Whitney $U$ test. (C) Small intestine lesions, $18 \mathrm{~h}$ after Indo administration. We stained formalin-fixed paraffin sections with hematoxylin and eosin, anti-F4/80 antibody (AbD Serotec, Kidlington, United Kingdom) for macrophages, and anti-Ly6G antibody (BD Biosciences, San Jose, CA, United States) for neutrophils. Bar $=50 \mu \mathrm{m}$.

family and the family Streptococcaceae were highly abundant in G3KO mice (Supplementary Figure 1).

Pathogenic or protective roles of individual bacteria in NSAID-induced small intestinal ulcers are not clear, although

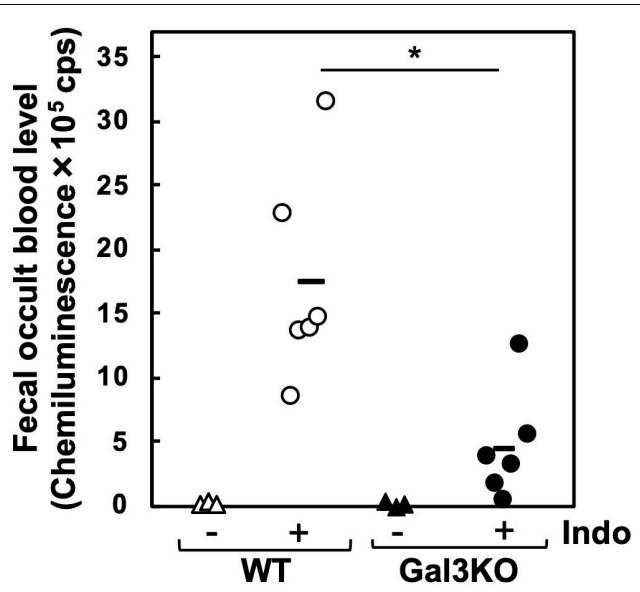

FIGURE 3 | Fecal occult blood (FOB) of Indo-administered mice. FOB levels were higher in Indo-administered WT mice (O) than in Gal3KO mice (•). Without Indo administration, FOB was not detectable in WT mice $(\Delta)$ or Gal3KO mice $(\boldsymbol{\Lambda})$. + Indo groups, $n=7$; - Ind groups, $n=3$. ${ }^{*} P<0.05$ WT+Indo versus Gal3KO+Indo by the Student $t$-test; count per second. FOB level was determined as described previously (43). Feces were suspended in distilled water and centrifuged at $12,000 \times g$. The supernatant was mixed with the luminol reagent (FUJIFILM Wako), and then chemiluminescence was measured by a luminometer (Wallac ARVO SX 1420 multilabel counter, PerkinElmer, Waltham, MA, United States).

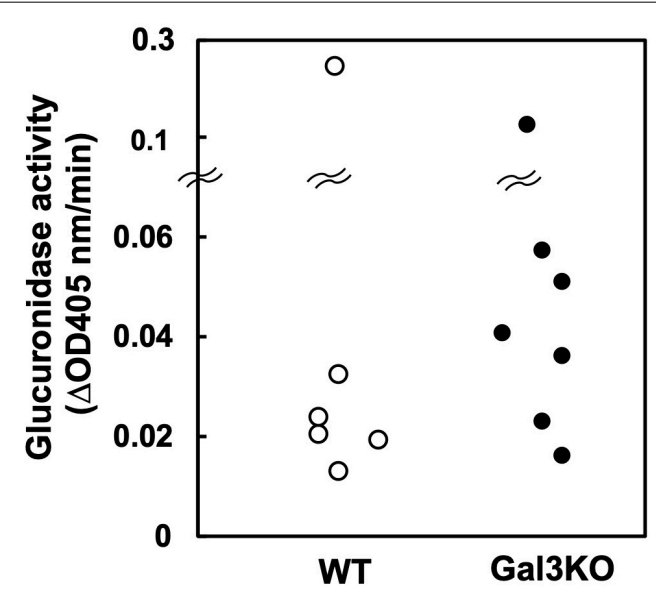

FIGURE 4 | Glucuronidase activity of fecal bacteria. Fecal suspension from naiive WT $(O)$ and Gal3KO mice $(\bullet)$ were prepared $(n=7)$. To determine $\beta$-glucuronidase activities, we incubated $20 \mu \mathrm{L}$ of the fecal suspension with $180 \mu \mathrm{L}$ of the reaction mixture containing $1 \mathrm{mM}$

p-nitrophenyl- $\beta$-D-glucuronide, $50 \mathrm{mM} \mathrm{HEPES}-\mathrm{HCl}(\mathrm{pH} 7.4)$, and $37.2 \mathrm{mM}$ 2-mercaptoethanol at $37^{\circ} \mathrm{C}$ for $30 \mathrm{~min}$. The optical density (OD) was measured at $405 \mathrm{~nm}$ every $5 \mathrm{~min}$ (24). $\triangle \mathrm{OD}$ : OD changes per $1 \mathrm{~min}$.

treatment with lactic acid-generating bacteria, including Lactobacillus, has been shown to change the intestinal microbiota, reducing intestinal ulcers $(27,28)$. To determine the roles of the intestinal microbiota, we first attempted to use co-housing of WT and Gal3KO mice as a means to transfer the microbiota, since cohousing has been shown to efficiently merge/exchange the fecal microbiota of the two mouse strains (29). Nevertheless, we found 
A

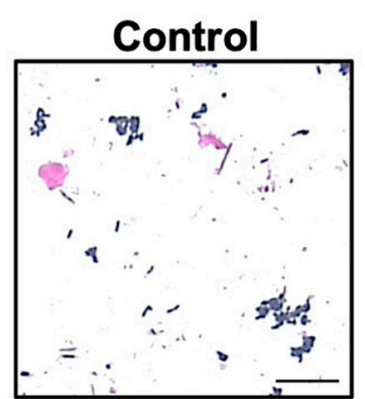

B

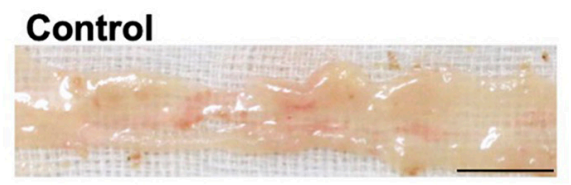

Polymyxin B

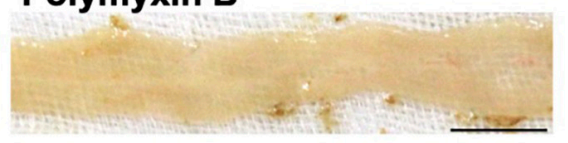

Neomycin

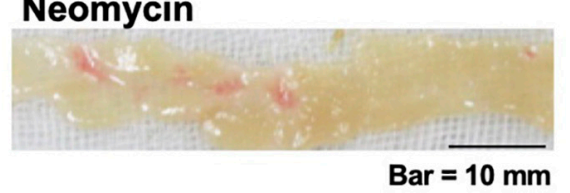

Polymyxin B

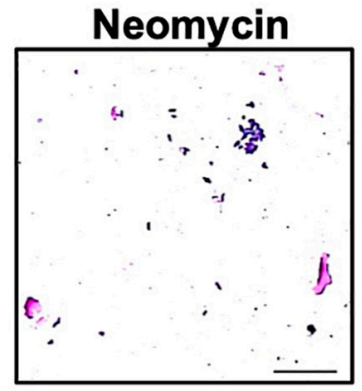

Bar $=20 \mu \mathrm{m}$

C

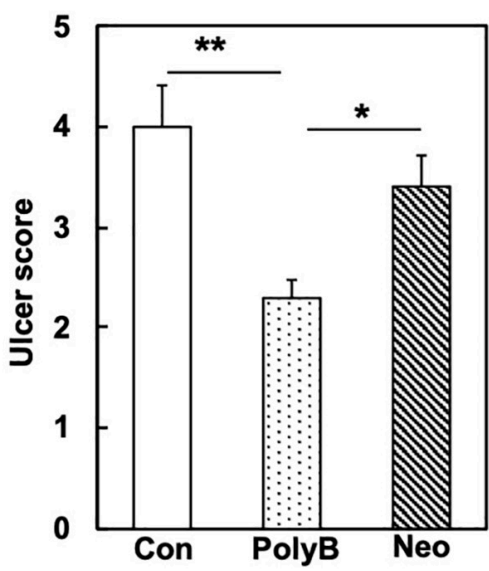

FIGURE 5 | Small intestinal microbial reduction by antibiotics and Indo-induced intestinal ulcers in WT mice. We treated WT mice with unabsorbable antibiotics, polymyxin B (PolyB, $0.5 \mathrm{mg} / \mathrm{mL}$, Pfizer Inc., New York, NY, United States) or neomycin (Neo, $1 \mathrm{mg} / \mathrm{mL}$, Thermo Fisher Scientific, Waltham, MA, United States) in drinking water. (A) On day 6, we killed mice and harvested small intestinal contents. We prepared suspensions of the contents in water and conducted Gram staining (FUJIFILM Wako). (B) On day 6, Indo was administrated, and $18 \mathrm{~h}$ later, we harvested, rinsed the small intestine, and took macroscopic images $(n=5)$. Representative images of the jejunum. Bar $=10 \mathrm{~mm}$. (C) PolyB-treated mice had lower ulcer scores than the control (Con) and Neo-treated mice. Ulcer severities were determined by the modified ulcer scores. ${ }^{\star *} P<0.01 ;{ }^{*} P<0.05$ by the ANOVA with post hoc Tukey test.

that there were significant differences in the small intestinal microbiota between WT and Gal3KO mice following co-housing. Thus, in our experimental system, fecal transplantation or coprophagy cannot be used for the equalization of the small intestinal microbiota.

Thus, to determine the extent of which intestinal microbiota could affect Indo-induced ulcers, we used an alternative protocol, treating mice with either the bactericidal antibiotic polymyxin B (PolyB) or bacteriostatic antibiotic neomycin dissolved in drinking water for 6 days before induction of ulcers (30). We confirmed the effects of the two antibiotics on the intestinal bacteria by conducting Gram staining of small intestinal contents harvested from the WT mice treated with the antibiotic, compared with those from the untreated control mice. In the control mice without antibiotics treatment, the majority of the bacteria were Gram-positive bacilli. We observed substantial reductions of small intestinal bacteria in the PolyB-treated group, although the effect of neomycin was limited (Figure 5A). Then, we assessed the levels of ulceration in the mice treated with each antibiotic. We found that ulceration of the PolyBtreated group was less severe than the control group. On the other hand, the neomycin-treated group had a mild decrease in ulceration compared with the control group (Figures 5B,C). Thus, our results further support the role of the gut microbiota in NSAID-induced ulcers, reported by other research groups who demonstrated the suppression of NSAID-induced intestinal ulcers by treatment with antibiotics including ampicillin and aztreonam $(24,25)$.

In the above experiments, we found that the amount of bacterial DNA in the jejunum contents from PolyB-treated mice decreased in $60 \%$ of that from untreated control mice, suggesting that the reduction of bacteria could contribute to the decrease in ulceration of PolyB-treated mice. Since the changes in bacterial composition can also affect the NSAIDinduced ulcers, using 16S rRNA sequencing of bacterial DNA, we compared the microbiomes in the jejunum contents between the PolyB-treated and control groups, using principal component analysis (Supplementary Figure 2) and alpha diversity indexes (Supplementary Figures 2, 3). Although there were no significant differences in alpha diversity of the microbiomes between the two groups (Supplementary Figure 3), PCA clearly separated the samples from the two groups into distinct 


\section{A \\ +Indo}

\section{Clod +Indo}

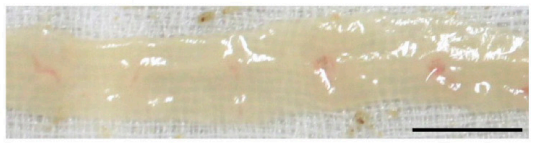

$\operatorname{Bar}=10 \mathrm{~mm}$

B

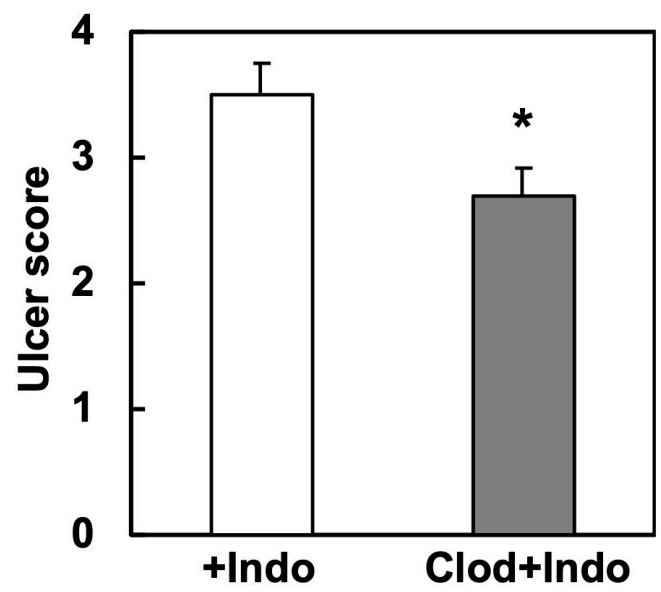

FIGURE 6 | Clodronate injection and Indo-induced intestinal ulcers in WT mice. We injected clodronate (Clod) intraperitoneally into WT mice. The next day, we administered Indo and killed mice $18 \mathrm{~h}$ later. We harvested and rinsed the small intestines, and took macroscopic images $(n=5)$. (A) Representative images of the jejunum from Indo-administered mice without Clod treatment (+Indo) showed more severe ulcers than mice treated with Clod (Clod+Indo). Bar $=10 \mathrm{~mm}$. (B) Ulcer severities were determined by the modified ulcer scores. ${ }^{*} P<0.05$ by the Mann-Whitney $U$ test.

populations (Supplementary Figure 2A), where there were significant differences in principal component (PC) 1 values between the two groups $(P<0.01$, Supplementary Figure 2B). Factor loading for PC1 showed that a decrease in the family Bacteroidaceae (order Bacteroidetes, Gram-negative) and an increase in the family Desulfovibrionaceae (order Proteobacteria, Gram-negative) correlated to PC1 values.

These results were consistent with the previous findings that Gram-negative bacilli including some species of Bacteroidetes were susceptible to PolyB (31), but some species of Desulfovibrionaceae had low sensitivity to PolyB (32). Previously, the high abundance (33-35) and the colitogenic property (36) of Bacteroidaceae have been reported in animal models of colitis. Bacteroidaceae can also enhance the immune reaction by activating dendritic cells (35). On the other hand, Desulfovibrionaceae has been reported to decrease in the feces of the DSS-induced colitis model and seemed to have protective effects against the inflammation (37). Desulfovibrionaceae can also produce hydrogen sulfide $\left(\mathrm{H}_{2} \mathrm{~S}\right)$, which plays roles in vasodilation and anti-inflammation in the gut (38). Thus, the changes in bacterial composition by PolyB treatment could be beneficial in the NSAID-induced ulcers.

\section{Role of Macrophages in NSAID-Induced Ulcers}

Since the Gal3 expression in macrophages has been reported to potentiate immune responses, we tested whether macrophage depletion could attenuate Indo-induced ulcers. Since clodronate, particularly its liposome-encapsulated form, has been used to deplete macrophages, we injected liposome-encapsulated clodronate, MacrokillerV300 (Cosmo Bio Co., Ltd., Tokyo, Japan), into mice. As observed by others, however, Macrokiller treatment alone caused severe body weight loss and diarrhea in the mice; we were unable to conduct an additional injection of Indo. Since the injection of clodronate itself $(30 \mathrm{mg} / \mathrm{kg}$, peritoneally, Tokyo Chemical Industry Co., Ltd., Tokyo, Japan) did not result in body weight changes or diarrhea, we treated mice with clodronate, instead of Macrokiller, 1 day before Indo administration. We found that the clodronate injection reduced the ulcer levels, mildly but significantly (Figure 6).

Since the number of small intestinal macrophages quantified by flow cytometry did not differ between the clodronate-injected versus control mice (data not shown), the mechanism by which clodronate suppressed the intestinal ulceration was unlikely due to the depletion, but suppression of macrophages functionally. Inhibition of macrophage-lineage cells by clodronate, a first-generation bisphosphonate, has been demonstrated. Experimentally, clodronate inhibits expression of tumor necrosis factor (TNF)- $\alpha$ and interleukin (IL)-6 in macrophages (39), suppressing inflammation. Clinically, clodronate has been prescribed for osteoporosis $(32,33)$; the pharmacological mechanism of clodronate is to inhibit the osteoclast activity with no macrophage-killing activity. Although clodronate has been shown not to suppress neutrophils directly (39), clodronate may affect neutrophils that infiltrate into Indoinduced ulcers indirectly; in theory, extracellular Gal3 released from macrophages could promote neutrophil functions $(9,10)$.

\section{DISCUSSION}

In this study, we found that Indo-induced ulcers were less severe in Gal3KO mice than in WT mice. We propose two mechanisms by which Gal3 could exacerbate Indo-induced intestinal ulcers; (1) the Gal3 expression in the small intestinal epithelia alters the bacterial population in the jejunum, enhancing mucosal bacterial invasion; and (2) the Gal3 expression in activated macrophages enhances inflammation. Consistently, we found that suppression of Indo-induced intestinal ulcers in WT mice seemed to be associated with both (1) gut microbial reduction and alteration by antibiotics treatment and (2) macrophage suppression by clodronate treatment. However, the levels of the ulcer suppression by the two treatments were not comparable to that of Gal3 KO mice (average ulcer scores: control WT group, 
3 to 4; antibiotics WT group, 2.3; clodronate WT group, 2.7; and Gal3KO group, 0.4) (Figures 2, 5, 6). Thus, our two proposed mechanisms may be closely related, and not mutually exclusive; for example, the enhanced bacterial invasion could activate macrophages. Although probiotics including Lactobacillus as well as macrophage inhibitors including clodronate can be useful to suppress the ulcers to some extent, Gal3 inhibition in both intestinal epithelia and macrophages is ideal for more efficient suppression of NSAID-induced small intestinal ulcers.

Galectin-3 affects numerous biological processes and has been suggested to play either beneficial or detrimental roles in several chronic diseases: amelioration of microbial infection $(9,33)$ and kidney function (40); exacerbation of atherosclerosis $(35,36)$, lung and renal fibrosis $(14,37)$, non-alcoholic steatohepatitis (NASH) (16, 38), and cancers $(17,39)$. Since most of the conditions listed above are associated with chronic diseases, it will be possible to minimize the adverse effects by prophylactic Gal3 inhibition for NSAID-induced small intestinal ulcers, if Gal3 inhibitors are used only for the short term. Indeed, galectin inhibitors, which inhibit Gal3 predominantly and Gal1 partially, have already been shown to be safe and applicable in several disease conditions, clinically and experimentally. Recently, TD139 also known as GB0139 has attracted attention as a highly specific Gal3/Gal1 inhibitor: the affinity of TD139 for Gal3 with $K_{d}=14 \mathrm{nM}$ and for Gal1 with $K_{d}=10 \mathrm{nM}$, but low for galectins $2,4,7,8$, or 9 (41). Currently, TD139 is used in a clinical trial for idiopathic pulmonary fibrosis (IPF). After successful results of Phase I/II trials which assessed the safety and tolerability in healthy volunteers and patients with IPF, Phase IIb clinical trial of TD139 treatment is now enrolling up to 450 eligible IPF patients at sites across the United States, Canada, Europe, United Kingdom, and Israel.

\section{CONCLUSION}

We demonstrated that inhibition of Gal3 could be a therapeutic strategy in NSAID-induced intestinal ulcers. Although targeting this molecule may cause several unexpected outcomes because of its variety of functions, Gal3 inhibitors, particularly for its short-term use, have already been shown to be safe, clinically and experimentally. The future invention of the Gal3 inhibitor that can target only a specific organ or cell type should be safe and effective to treat a variety of disease conditions; an intestinespecific Gal3 inhibitor could reduce NSAID-induced intestinal ulcers without adverse effects.

\section{MATERIALS AND METHODS}

\section{Mice}

We purchased CD1 mice from Charles River Laboratories Japan (Yokohama, Japan). The generation of Gal3KO mice was described previously (12). The animal experiments were approved by the Institutional Animal Care and Use Committee of Kindai University (Osaka, Japan) and performed in accordance with the institutional guidelines.

\section{Immunological Staining, PAS Staining, and HE Staining}

We made 4 - $\mu$ m-thick tissue sections. Immunohistochemistry (IHC) was performed by the standard procedure using Histofine SAB-PO kit (Nichirei Biosciences; Tokyo, Japan) and 3,3'-diaminobenzidine (DAB). Polyclonal antibodies used for IHC were as follows: anti-Gal3 (BioLegend, San Diego, CA, United States), anti-F4/80 (AbD Serotec, Kidlington, United Kingdom), and anti-Ly6/G (BD Biosciences, San Jose, CA, United States). PAS and hematoxylin and eosin (H\&E) staining were performed by the standard procedures.

\section{Induction and Assessment of Indo-Induced Small Intestinal Ulcers}

Indo (FUJIFILM Wako, Osaka, Japan) was dissolved in a $0.5 \%$ $\mathrm{NaHCO}_{3}$ solution and administrated to non-fasted mice by oral gavage. Control mice were administered a $0.5 \% \mathrm{NaHCO}_{3}$ solution alone. We determined the Indo dosage $(5 \mathrm{mg} / \mathrm{kg}$ body weight) by "Human equivalent dose calculation" (42). At $18 \mathrm{~h}$ after the injection, we collected feces, took blood from the right ventricle, and perfused the mice with phosphate-buffered saline (PBS) from the left ventricle. We harvested and rinsed the small intestines with PBS, opened along the anti-mesenteric side and took macroscopic images with a scale by a digital camera (Canon, Tokyo, Japan). Then, tissue pieces were fixed in formalin and embedded in paraffin for histological examinations.

The ulcer severity was evaluated using the ulcer score (23) with modification. The macroscopic ulceration areas were captured and quantified by an ImageJ software (NIH, Bethesda, MD, United States) and summed per jejunum. The entire jejunum areas were similar in all mice examined and were around 1,200 $\mathrm{mm}^{2}$. We used the modified ulcer score as follows: $0=$ no lesion, $1=$ less than $5 \mathrm{~mm}^{2}, 2=5 \sim 20 \mathrm{~mm}^{2}, 3=21 \sim 40 \mathrm{~mm}^{2}$, $4=41 \sim 70 \mathrm{~mm}^{2}$, and $5=$ more than $71 \mathrm{~mm}^{2}$.

Fecal occult blood level was determined as described previously (43). Feces were suspended in distilled water and centrifuged at $12,000 \times g$. The supernatant was mixed with a luminol reagent (FUJIFILM Wako), and then chemiluminescence was measured by a luminometer (Wallac ARVO SX 1420 multilabel counter, PerkinElmer, Waltham, MA, United States).

\section{$\beta$-Glucuronidase Activities of Feces}

To determine $\beta$-glucuronidase activities, we mixed $20 \mu \mathrm{L}$ of the fecal suspension with $180 \mu \mathrm{L}$ of the reaction mixture containing $1 \mathrm{mM} p$-nitrophenyl- $\beta$-D-glucuronide (FUJIFILM Wako), $50 \mathrm{mM}$ HEPES-HCl ( $\mathrm{pH} 7.4$ ), and $37.2 \mathrm{mM}$ 2mercaptoethanol (24). We incubated the mixture at $37^{\circ} \mathrm{C}$ and measured its optical density (OD) at $405 \mathrm{~nm}$, every $5 \mathrm{~min}$ between 10 - and 30-min incubation times. We calculated the average OD change per min.

\section{Antibiotics Treatment and Gram-Staining}

We treated WT mice with an unabsorbable antibiotic, polymyxin B (PolyB, 0.5 mg/mL, Pfizer Inc., New York, NY, United States) or neomycin (Neo, $1 \mathrm{mg} / \mathrm{mL}$, Thermo Fisher Scientific, Waltham, MA, United States) in drinking water ( $n=9$ per group). On day 6 , we killed four mice in each treatment group and harvested small 
intestinal contents. We prepared suspensions of the contents in water and conducted Gram staining (FUJIFILM Wako). Using the remaining five mice in each treatment group, we administered Indo and assessed the ulcer levels, using the ulcer score.

\section{Effect of Clodronate Injection on Indo-Induces Ulcers}

One day before Indo administration, we injected clodronate (30 mg/kg body weight, FUJIFILM Wako) intraperitoneally into WT mice. Then, we administered Indo and assessed the ulcer levels, using the ulcer score.

\section{DATA AVAILABILITY STATEMENT}

The raw data supporting the conclusions of this article will be made available by the authors, without undue reservation.

\section{ETHICS STATEMENT}

The animal study was reviewed and approved by the Institutional Animal Care and Use Committee of Kindai University.

\section{AUTHOR CONTRIBUTIONS}

A-MP designed and conducted the experiments. IT supervised the project. A-MP and IT wrote the manuscript. SK performed the microbiome analysis. FS, SO, MF, DKH, and F-TL conceived

\section{REFERENCES}

1. Bjarnason I, Hayllar J, MacPherson AJ, Russell AS. Side effects of nonsteroidal anti-inflammatory drugs on the small and large intestine in humans. Gastroenterology. (1993) 104:1832-47. doi: 10.1016/0016-5085(93)90 667-2

2. Lewis JD, Bilker WB, Brensinger C, Farrar JT, Strom BL. Hospitalization and mortality rates from peptic ulcer disease and GI bleeding in the 1990s: relationship to sales of nonsteroidal anti-inflammatory drugs and acid suppression medications. Am J Gastroenterol. (2002) 97:2540-9. doi: 10.1111/ j.1572-0241.2002.06037.x

3. Davies NM, Saleh JY, Skjodt NM. Detection and prevention of NSAIDinduced enteropathy. J Pharm Pharm Sci. (2000) 3:137-55.

4. Barondes SH, Cooper DN, Gitt MA, Leffler H. Galectins. Structure and function of a large family of animal lectins. J Biol Chem. (1994) 269:20807-10.

5. Sundblad V, Croci DO, Rabinovich GA. Regulated expression of galectin3, a multifunctional glycan-binding protein, in haematopoietic and nonhaematopoietic tissues. Histol Histopathol. (2011) 26:247-65. doi: 10.14670/ HH-26.247

6. Hsu DK, Yang RY, Pan Z, Yu L, Salomon DR, Fung-Leung WP, et al. Targeted disruption of the galectin-3 gene results in attenuated peritoneal inflammatory responses. Am J Pathol. (2000) 156:1073-83. doi: 10.1016/S0002-9440(10) 64975-9

7. Beatty WL, Rhoades ER, Hsu DK, Liu F-T, Russell DG. Association of a macrophage galactoside-binding protein with Mycobacterium-containing phagosomes. Cell Microbiol. (2002) 4:167-76. doi: 10.1046/j.1462-5822.2002. 00183.x

8. Sano H, Hsu DK, Apgar JR, Yu L, Sharma BB, Kuwabara I, et al. Critical role of galectin-3 in phagocytosis by macrophages. J Clin Invest. (2003) 112:389-97. doi: $10.1172 /$ JCI17592 and conducted some experiments. All authors read and approved the final manuscript.

\section{FUNDING}

This work was supported by grants from the National Institute of General Medical Sciences, COBRE Grant (P30-GM110703), the Japan Society for the Promotion of Science [JSPS, Grants-inAid for Scientific Research-KAKENHI, 20K07455 (IT), 20K07433 (FS), 15K08975 (A-MP), and 18K07379 (A-MP)], and the Novartis Pharma Research Grants (A-MP and IT). The funder was not involved in the study design, collection, analysis, interpretation of data, the writing of this article or the decision to submit it for publication.

\section{ACKNOWLEDGMENTS}

We thank Namie Sakiyama, Aoshi Katsuki, Yumina Nakamura, Felicia Lindeberg, and Erika Pedio for excellent technical assistance. We also thank Dr. Osamu Yoshie for helpful comments.

\section{SUPPLEMENTARY MATERIAL}

The Supplementary Material for this article can be found online at: https://www.frontiersin.org/articles/10.3389/fimmu. 2020.550366/full\#supplementary-material

9. Farnworth SL, Henderson NC, Mackinnon AC, Atkinson KM, Wilkinson T, Dhaliwal K, et al. Galectin-3 reduces the severity of pneumococcal pneumonia by augmenting neutrophil function. Am J Pathol. (2008) 172:395-405. doi: 10.2353/ajpath.2008.070870

10. Sato S, Ouellet N, Pelletier I, Simard M, Rancourt A, Bergeron MG. Role of galectin-3 as an adhesion molecule for neutrophil extravasation during streptococcal pneumonia. J Immunol. (2002) 168:1813-22. doi: 10.4049/ jimmunol.168.4.1813

11. Fermino ML, Polli CD, Toledo KA, Liu F-T, Hsu DK, Roque-Barreira MC, et al. LPS-induced galectin-3 oligomerization results in enhancement of neutrophil activation. PLoS One. (2011) 6:e26004. doi: 10.1371/journal.pone.0026004

12. Park A-M, Hagiwara S, Hsu DK, Liu F-T, Yoshie O. Galectin-3 plays an important role in innate immunity to gastric infection by Helicobacter pylori. Infect Immun. (2016) 84:1184-93. doi: 10.1128/IAI.01299-15

13. Kohatsu L, Hsu DK, Jegalian AG, Liu F-T, Baum LG. Galectin-3 induces death of Candida species expressing specific beta-1,2-linked mannans. J Immunol. (2006) 177:4718-26. doi: 10.4049/jimmunol.177.7.4718

14. Mackinnon AC, Gibbons MA, Farnworth SL, Leffler H, Nilsson UJ, Delaine $\mathrm{T}$, et al. Regulation of transforming growth factor- $\beta 1$-driven lung fibrosis by galectin-3. Am J Respir Crit Care Med. (2012) 185:537-46. doi: 10.1164/rccm. 201106-0965OC

15. Li L, Li J, Gao J. Functions of galectin-3 and its role in fibrotic diseases. $J$ Pharmacol Exp Ther. (2014) 351:336-43. doi: 10.1124/jpet.114.218370

16. Harrison SA, Marri SR, Chalasani N, Kohli R, Aronstein W, Thompson GA, et al. Randomised clinical study: GR-MD-02, a galectin-3 inhibitor, vs. placebo in patients having non-alcoholic steatohepatitis with advanced fibrosis. Aliment Pharmacol Ther. (2016) 44:1183-98. doi: 10.1111/apt.13816

17. Eliaz I. The role of galectin-3 as a marker of cancer and inflammation in a stage IV ovarian cancer patient with underlying pro-inflammatory comorbidities. Case Rep Oncol. (2013) 6:343-9. doi: 10.1159/000353574 
18. Dong R, Zhang M, Hu Q, Zheng S, Soh A, Zheng Y, et al. Galectin-3 as a novel biomarker for disease diagnosis and a target for therapy (Review). Int J Mol Med. (2018) 41:599-614. doi: 10.3892/ijmm.2017.3311

19. Takeuchi K, Tanaka A, Ohno R, Yokota A. Role of COX inhibition in pathogenesis of NSAID-induced small intestinal damage. J Physiol Pharmacol. (2003) 54(Suppl. 4):165-82.

20. Takeuchi K, Satoh H. NSAID-induced small intestinal damage-roles of various pathogenic factors. Digestion. (2015) 91:218-32. doi: 10.1159/000374106

21. Delacour D, Koch A, Ackermann W, Eude-Le Parco I, Elsässer H-P, Poirier F, et al. Loss of galectin-3 impairs membrane polarisation of mouse enterocytes in vivo. J Cell Sci. (2008) 121:458-65. doi: 10.1242/jcs.020800

22. Weissenborn U, Maedge S, Buettner D, Sewing KF. Indometacin-induced gastrointestinal lesions in relation to tissue concentration, food intake and bacterial invasion in the rat. Pharmacology. (1985) 30:32-9. doi: 10.1159/ 000138047

23. Fukumoto K, Naito Y, Takagi T, Yamada S, Horie R, Inoue K, et al. Role of tumor necrosis factor- $\alpha$ in the pathogenesis of indomethacin-induced small intestinal injury in mice. Int J Mol Med. (2011) 27:353-9. doi: 10.3892/ijmm. 2011.602

24. Dabek M, McCrae SI, Stevens VJ, Duncan SH, Louis P. Distribution of betaglucosidase and beta-glucuronidase activity and of beta-glucuronidase gene gus in human colonic bacteria. FEMS Microbiol Ecol. (2008) 66:487-95. doi: 10.1111/j.1574-6941.2008.00520.x

25. Saitta KS, Zhang C, Lee KK, Fujimoto K, Redinbo MR, Boelsterli UA. Bacterial $\beta$-glucuronidase inhibition protects mice against enteropathy induced by indomethacin, ketoprofen or diclofenac: mode of action and pharmacokinetics. Xenobiotica. (2014) 44:28-35. doi: 10.3109/00498254.2013. 811314

26. Watanabe T, Higuchi K, Kobata A, Nishio H, Tanigawa T, Shiba M, et al. Non-steroidal anti-inflammatory drug-induced small intestinal damage is Toll-like receptor 4 dependent. Gut. (2008) 57:181-7. doi: 10.1136/gut.2007. 125963

27. Montalto M, Gallo A, Curigliano V, D’Onofrio F, Santoro L, Covino M, et al. Clinical trial: the effects of a probiotic mixture on non-steroidal antiinflammatory drug enteropathy - a randomized, double-blind, cross-over, placebo-controlled study. Aliment Pharmacol Ther. (2010) 32:209-14. doi: 10.1111/j.1365-2036.2010.04324.x

28. Konaka A, Kato S, Tanaka A, Kunikata T, Korolkiewicz R, Takeuchi K. Roles of enterobacteria, nitric oxide and neutrophil in pathogenesis of indomethacininduced small intestinal lesions in rats. Pharmacol Res. (1999) 40:517-24. doi: 10.1006/phrs.1999.0550

29. McCoy KD, Geuking MB, Ronchi F. Gut microbiome standardization in control and experimental mice. Curr Protoc Immunol. (2017) 117:23.1.123.1.13. doi: $10.1002 /$ cpim. 25

30. Emmelot $\mathrm{CH}$, van der Waaij $\mathrm{D}$. The dose at which neomycin and polymyxin $\mathrm{B}$ can be applied for selective decontamination of the digestive tract in mice. J Hyg (Lond). (1980) 84:331-40. doi: 10.1017/s002217240002 6851

31. Mitsuoka T, Terada A, Watanabe K, Uchida K. Bacteroides multiacidus, a new species from the feces of humans and pigs. Int J Syst Bacteriol. (1974) 24:35-41. doi: 10.1099/00207713-24-1-35

32. Jayaraman A, Mansfeld FB, Wood TK. Inhibiting sulfate-reducing bacteria in biofilms by expressing the antimicrobial peptides indolicidin and bactenecin.
J Ind Microbiol Biotechnol. (1999) 22:167-75. doi: 10.1038/sj.jim.290 0627

33. Osaka T, Moriyama E, Arai S, Date Y, Yagi J, Kikuchi J, et al. Meta-analysis of fecal microbiota and metabolites in experimental colitic mice during the inflammatory and healing phases. Nutrients. (2017) 9:1329. doi: 10.3390/ nu9121329

34. Huang Y-L, Chassard C, Hausmann M, von Itzstein M, Hennet T. Sialic acid catabolism drives intestinal inflammation and microbial dysbiosis in mice. Nat Commun. (2015) 6:8141. doi: 10.1038/ncomms9141

35. Grabinger T, Glaus Garzon JF, Hausmann M, Geirnaert A, Lacroix C, Hennet T. Alleviation of intestinal inflammation by oral supplementation with 2fucosyllactose in mice. Front Microbiol. (2019) 10:1385. doi: 10.3389/fmicb. 2019.01385

36. Bloom SM, Bijanki VN, Nava GM, Sun L, Malvin NP, Donermeyer DL, et al. Commensal Bacteroides species induce colitis in host-genotype-specific fashion in a mouse model of inflammatory bowel disease. Cell Host Microbe. (2011) 9:390-403. doi: 10.1016/j.chom.2011.04.009

37. Darnaud M, Dos Santos A, Gonzalez P, Augui S, Lacoste C, Desterke C, et al. Enteric delivery of regenerating family member 3 alpha alters the intestinal microbiota and controls inflammation in mice with colitis. Gastroenterology. (2018) 154:1009-23.e14. doi: 10.1053/j.gastro.2017.11.003

38. Magierowski M, Jasnos K, Kwiecien S, Drozdowicz D, Surmiak M, Strzalka $M$, et al. Endogenous prostaglandins and afferent sensory nerves in gastroprotective effect of hydrogen sulfide against stress-induced gastric lesions. PLoS One. (2015) 10:e0118972. doi: 10.1371/journal.pone.0118972

39. Kato Y, Hiasa M, Ichikawa R, Hasuzawa N, Kadowaki A, Iwatsuki K, et al. Identification of a vesicular ATP release inhibitor for the treatment of neuropathic and inflammatory pain. Proc Natl Acad Sci USA. (2017) 114:E6297-305. doi: 10.1073/pnas.1704847114

40. Bichara M, Attmane-Elakeb A, Brown D, Essig M, Karim Z, Muffat-Joly M, et al. Exploring the role of galectin 3 in kidney function: a genetic approach. Glycobiology. (2006) 16:36-45. doi: 10.1093/glycob/cwj035

41. Chan Y-C, Lin H-Y, Tu Z, Kuo Y-H, Hsu S-TD, Lin C-H. Dissecting the structure-activity relationship of galectin-ligand interactions. Int J Mol Sci. (2018) 19:392. doi: 10.3390/ijms19020392

42. Nair AB, Jacob S. A simple practice guide for dose conversion between animals and human. J Basic Clin Pharm. (2016) 7:27-31. doi: 10.4103/0976-0105. 177703

43. Park A-M, Tsunoda I. Forensic luminol reaction for detecting fecal occult blood in experimental mice. BioTechniques. (2018) 65:227-30. doi: 10.2144/ btn-2018-0017

Conflict of Interest: The authors declare that the research was conducted in the absence of any commercial or financial relationships that could be construed as a potential conflict of interest.

Copyright (c) 2020 Park, Khadka, Sato, Omura, Fujita, Hsu, Liu and Tsunoda. This is an open-access article distributed under the terms of the Creative Commons Attribution License (CC BY). The use, distribution or reproduction in other forums is permitted, provided the original author(s) and the copyright owner(s) are credited and that the original publication in this journal is cited, in accordance with accepted academic practice. No use, distribution or reproduction is permitted which does not comply with these terms. 